\title{
NORMAS Y PERCEPCIONES SOBRE CARRERA ACADÉMICA EN CHILE
}

\section{Introducción}

La profesión académica ha sido escasamente estudiada fuera de los países desarrollados. La bibliografía principal de circulación internacional, en lo que concierne a América Latina, se reduce al trabajo de Daniel C. Levy (1986), que incluye al profesorado como objeto de estudio pero trata más bien sobre la educación superior privada, los volúmenes editados por Philip G. Altbach $(1994,2003)$ sobre esta profesión en perspectiva internacional comparada, el clásico de Manuel Gil-Antón (1994) sobre la profesión académica en México y diversos trabajos posteriores del mismo autor, y el libro editado el año pasado por Nelly Stromquist (2007) que incluye casos de Brasil, Perú, Argentina, y México, entre otros países. Además, existen estudios de caso de universidades en particular, especialmente en México (Parra Sandoval, 2004; Chavoya Peña, 2001; García Salord, 2001, Grediaga Kuri, 2001; Landesmann, 2001; Villa Lever, 2001). Aunque es evidente la falta de desarrollo en estudios sobre educación superior en Chile y en América Latina, existe cierta tradición de investigaciones sobre activismo político estudiantil, sobre relación entre universidades y poder político, y sobre políticas de financiamiento, mientras que acerca del profesorado universitario, en cuanto actores y objeto de estudio, la investigación ha tenido mucha menos visibilidad.

La profesión o carrera académica implica el ejercicio de una actividad profesional dentro de una estructura organizacional particular, como es el caso de las instituciones universitarias, en las cuales las racionalidades de funcionamiento remiten no sólo a relaciones puramente académicas, sino que a lógicas de poder, de estructuración en torno a un sistema de prestigio y de administración de los bienes académicos. 
La sociología de la ciencia enseña que los logros científicos y el avance en la carrera académica no dependen sólo de la dedicación al trabajo y el rigor con que se practica el método científico, y que la experiencia de ser investigador no es homogénea a través de diversos grupos demográficos. Siguiendo la hipótesis de las ventajas acumulativas, o "efecto Mateo" de Merton (1973), según la cual las ventajas tempranas en la carrera científica se traducen en mayores ventajas a lo largo del tiempo, la literatura internacional se ha concentrado en el sexo, la raza y la edad como condicionantes de las oportunidades de los científicos de avanzar en sus carreras, de su productividad y visibilidad, y del acceso a recursos (Cole \& Cole, 1973; Allison \& Stewart, 1974; Zuckerman, Cole \& Bruer, 1991).

Entre las evidencias mundiales destaca el hecho de que en gran parte de los países, hacia fines del siglo XX, el cuerpo académico seguía siendo dominado por los varones, y la mayoría de éstos era de mediana edad (entre los 40 y 50 años). Mientras casi la mayoría de los profesores estaban en posiciones de jornada completa, en algunos pocos países también fue posible encontrarlos como minorías en puestos de tiempo parcial (Altbach, 1994).

En el caso chileno se observan tendencias similares. Este artículo tiene por objeto presentar la forma en que la carrera académica ha tendido a converger en las universidades chilenas hacia un único modelo ideal de académico, centrado en la producción de investigación, no obstante lo cual subsisten en la trayectoria de la mayoría de los profesores universitarios chilenos otras "carreras académicas", ancladas en la docencia y en la gestión, que no se encuentran bien reflejadas en ese modelo y mantienen con él una posición subordinada.

Las reflexiones que aquí se ofrecen sobre la academia chilena están basadas en resultados preliminares y generales de mi estudio, actualmente en curso, "Normas y percepciones sobre carrera académica. Análisis del contexto institucional y de la experiencia del profesorado en universidades chilenas", patrocinado por el Consejo Superior de Educación (CSE). 


\section{Carrera académica y contexto institucional}

Las universidades comienzan a distinguirse en el mercado de la educación superior (ES) y la investigación les sirve para acreditarse a un nivel mayor. Por ello, obtienen incentivos para estimularla, lo que, por una parte, les otorga estatus dentro del sistema o mercado de la ES y, por otra, les sirve para atraer recursos, ya sea a través de la captación de más y mejores estudiantes o de fondos para la innovación e investigación.

Para lograr este reconocimiento las universidades necesitan un cuerpo de profesores con niveles altos de productividad académica, que les permita competir sobre la base de indicadores de evaluación ya establecidos como norma en el sistema global de ES del que Chile crecientemente forma parte, como son la adjudicación de fondos para realizar investigación y la publicación en revistas científicas. En el caso chileno, esto se traduce en los proyectos del Fondo Nacional de Desarrollo Científico y Tecnológico (FONDECYT) o del Fondo de Fomento al Desarrollo Científico y Tecnológico (FONDEF) y en la publicación de artículos en revistas ISI (Information Sciences Institute) o SciELO (Scientific Electronic Library Online).

La carrera académica establece claramente cuáles son los roles del profesorado. Y junto a las funciones de investigación está la docencia y las labores de administración. Así entendida, da forma al tipo de regulaciones bajo las que se regirá la estructura universitaria. Éstas se erigen como patrones y así son asumidas y reconocidas por los miembros que componen la comunidad de profesores.

Para establecer el origen de lo que se considera deseable para la carrera académica, sería necesario remitirse a la historia de las universidades. Allí se podría encontrar respuesta a cómo se estableció la diferencia entre el quehacer puramente docente que cumplía la universidad en sus orígenes y el investigativo que prevalece en el paradigma de la productividad científica en la sociedad actual. 
La Universidad de Berlín constituyó un hito en la historia moderna de las casas de estudios superiores, por cuanto introdujo la innovadora idea de unir por primera vez docencia e investigación. Sólo a partir del siglo XIX empiezan a surgir universidades que se organizan progresivamente en torno a la "racionalidad cognitiva", esto es, la producción de nuevos conocimientos mediante la investigación. En la universidad europea moderna los atributos de la excelencia no dependían de una función que ni siquiera existía, como es la producción institucionalizada de conocimiento a través de la investigación empírica, sino que estaban asociados más bien al ideal de profesor refinado o cultivado. El principal objetivo del cuerpo académico era desarrollar la personalidad culta de los estudiantes, más que brindarles herramientas para el desempeño de la profesión o la ciencia. Por tal motivo, lo que más se valoraba era el manejo acabado de lo más apreciado en la cultura (Brunner, 1994).

\section{En busca del perfil ideal}

Cabe preguntarse por qué las universidades instalan la idea de un modelo normativo ideal del "ser académico", que se establece en el actual funcionamiento de las universidades bajo la noción de "carrera académica". Cuando se hace referencia a un modelo normativo se alude a pautas, que se expresan institucionalmente a través de los reglamentos de carrera académica de cada universidad. Éstas dan el marco para entender lo que puede ser considerado óptimo o ideal dentro del trabajo universitario.

Lo anterior cobra mayor sentido toda vez que quienes trabajan hoy en las universidades son parte de un proceso de profesionalización de la carrera académica. En este contexto, el reglamento de dicha carrera establece los requisitos, criterios y procedimientos para la evaluación del desempeño de los profesores, a la vez que los distribuye jerárquicamente mediante rangos en el sistema de prestigio (y usualmente también de remuneraciones) de la institución. 
Lo interesante del fenómeno de la "profesionalización de la carrera académica" es que tiende a hacerla cada vez más homogénea dentro del mercado de las universidades. Los requisitos y criterios para evaluar el trabajo están siendo hoy más uniformes y lo que es un requisito en la academia chilena también lo es para la europea, norteamericana o brasileña. Esto es en parte efecto de la globalización y la simultaneidad de las comunicaciones, de las cuales la ciencia es el precursor más notable y de cuya influencia no ha escapado el sistema de ES.

En este escenario de mercados cada vez más definidos y regulados, los mejores profesores investigadores vienen a encarnar la figura del modelo ideal, representando las cualidades que éste impone. El modelo es socialmente construido por una comunidad científica autoritativa y requiere del consenso y reconocimiento de los miembros que conforman la comunidad académica para que tenga legitimidad. Por lo mismo, no ha sido el mismo en la historia de las universidades.

La hegemonía de un modelo da lugar a representaciones sobre lo que es ser un buen académico e implícitamente construye su opuesto. Dichas representaciones dan cuenta del ámbito cultural en el que se desenvuelve el trabajo académico y se vinculan con los símbolos asociados a lo valorado o subvalorado, estableciendo distinciones y jerarquías de poder al interior de las instituciones. Se instauran así normas, códigos de conducta (motivaciones personales) y modos de relacionarse entre los profesores.

Cuando se menciona la existencia del modelo ideal normativo como una construcción social situada históricamente se hace alusión al hecho de que existe una comunidad científica que define los criterios de excelencia en la producción de conocimientos y que, en el caso concreto de las universidades, hace equivalentes el paradigma de la productividad científica con los criterios fácilmente medibles posibilitados por la investigación. Se sitúa históricamente porque, como hemos señalado, no siempre este ideal ha estado asociado a la investigación. 
En la actualidad, el perfil ideal está claramente cuantificado: es posible medirlo y, por tanto, evaluarlo. Este hecho le da una gran legitimidad y preponderancia a la investigación científica. Por ello, el académico ideal es aquel que reúne -a juicio de la comunidad universitaria, avalado por el grupo de pares- las mejores cualidades encarnadas en la figura del investigador que produce conocimiento, da a conocer sus resultados y atrae recursos. Otra característica bien valorada es la formación de discípulos, traducida, por ejemplo, en número de tesis guiadas. En este caso se establecen incluso distinciones más finas entre el pre y el posgrado, y así está estipulado en la valoración al momento de las evaluaciones de desempeño, especialmente cuando se postula a una promoción o cambio a una jerarquía mayor dentro de la propia institución.

No obstante que este perfil esté claramente identificado y expresado en los reglamentos de carrera académica de las universidades, es posible observar criterios de evaluación difíciles de cuantificar pero que operan como distinciones más "refinadas", por ejemplo, haber obtenido reconocimiento internacional, típicamente exigible a los que postulan al cargo de profesor titular. Introducir estos elementos de carácter más bien cualitativo en la evaluación académica dirige la atención hacia las unidades organizacionales que actúan como comités de evaluación, los cuales, compuestos por académicos de los más altos rangos, insertos en un contexto internacional y atentos a las demandas de un sistema de educación superior mayor, se instalan como los guardianes y defensores del modelo ideal normativo del desarrollo de carrera académica.

En "Sociología de la Ciencia", Merton (1973) describe el cuarto rol de "guardián", junto con los de investigación, docencia y administración. Sostiene que este rol es básico para los sistemas de evaluación y de asignación de funciones y recursos en la ciencia. Estos guardianes de la ciencia evalúan el potencial y las limitaciones de los aspirantes a nuevas posiciones, afectando de ese modo la movilidad individual de los científicos y el lugar y distribución del personal a través del sistema. 
En el mundo académico existen ritos que dan sentido de pertenencia y afiliación a sus miembros. Estos son, por ejemplo, la obtención del grado de doctor, el ascenso a jerarquías de mayor prestigio, la adjudicación de fondos de investigación en concursos competitivos, la estadía de investigación en otra institución o la consecución de un cargo directivo relevante. Notablemente, casi todos ellos remiten a los hitos en la carrera de un profesor investigador.

\section{Carrera vs. carreras académicas}

Si bien el modelo ideal normativo "investigador" cada vez está más extendido e internalizado existen otras carreras "paralelas", más cercanas a un perfil docente o administrativo. En el sistema de prestigio esto último es lo que tiene menor valoración y es representado en el imaginario del trabajo académico como roles con menor importancia. La docencia no otorga el mismo prestigio que la investigación, pero mantiene una cierta legitimidad por su relevancia social y profesional.

Esta división del trabajo delimita los lugares por los cuales los académicos pueden transitar, y es frecuente encontrar, por ejemplo, una mayor concentración de la carga de trabajo docente en mujeres y jóvenes que están en los inicios de sus carreras; en general, en el conjunto de académicos que pertenecen a universidades cuyo perfil es más docente o que son parte de aquellas disciplinas más ligadas a carreras de "tiza y pizarrón" que a laboratorio.

Existen distintas maneras de entender la carrera académica según el punto de vista del agente, ya sea desde la perspectiva de los actores mismos del sistema -que en este caso corresponde al profesorado universitario- o desde la visión que establecen las propias instituciones universitarias.

Desde los actores, por ejemplo, se observa que dicha carrera se configura sobre la base de ciertos eventos o hitos por los que atraviesan cada uno de los académicos, estableciéndose trayectorias 
de trabajo con características específicas según la disciplina en la que se encuentren y la edad y el tipo de institución al que estén afiliados. Los eventos o hitos que configuran las trayectorias académicas pueden ser graficados de la siguiente manera:

Ingreso $\rightarrow$ desarrollo $\rightarrow$ promoción $\rightarrow$ permanencia $\rightarrow$ retiro

Desde las instituciones, la definición de la carrera académica se configura en torno a un contexto de regulación del quehacer del profesorado, estableciendo roles, responsabilidades, a la vez que un sistema de distribución en la estructura de prestigio y de jerarquías al interior de las instituciones. Estas regulaciones se erigen como normas y son asumidas y reconocidas por los miembros que componen la comunidad académica. Al igual que en la perspectiva del profesorado universitario, también se puede determinar una línea o trayectoria sobre cómo las instituciones establecen lo que es la carrera académica:

Requisitos $\rightarrow$ normas y procedimientos $\rightarrow$ evaluación de desempeño

En la perspectiva de los sujetos, el significado que adquiere la carrera académica está relacionado con la historia personal dentro de la institución y responde también a la cultura organizacional, las relaciones interpersonales y a la ética del trabajo académico en el contexto de la facultad, escuela o departamento.

Existen tantos modelos de carrera académica como historias de vida entre el profesorado universitario, ya que, según evidencias consignadas tanto en la literatura especializada como en la propia investigación en curso, no existe una única forma de ser académico y se observan diferencias entre las generaciones maduras y las más jóvenes, por ejemplo, o entre las ciencias puras y aplicadas y las humanidades o ciencias sociales, o entre hombres y mujeres.

Las generaciones jóvenes están socializadas bajo el "paradigma de la productividad científica" y tienen muy internalizado, como parte de las normas que orientan su trabajo, que es un requisito de entrada 
estar en posesión del grado de doctor o publicar en revistas indexadas. Por el contrario, las generaciones mayores o más antiguas configuraron su modo de ser académico en la propia práctica, especialmente en el caso de las ciencias sociales y humanas, en las cuales no es tan frecuente encontrar los doctorados y menos aún los posdoctorados.

En las ciencias puras y aplicadas, en cambio, es prácticamente una norma el estar en posesión de estos grados. Las carreras académicas de quienes se dedican a éstas se ubican en un lugar privilegiado, en tanto sus labores corresponden al modelo ideal normativo actual, basado en la mayor valoración de la investigación y las publicaciones. Esto se refuerza positivamente con la oferta de programas doctorales, la existencia de revistas especializadas donde publicar y la provisión de fondos para investigar, más abundantes en este grupo de disciplinas que en las ciencias sociales o las humanidades.

En el caso de las diferencias entre hombres y mujeres, las desventajas que enfrentan estas últimas están suficientemente documentadas en EE.UU. (Berg y Ferber, 1983; Stage and Maple, 1996; Kurtz-Costes et al., 2006 y los otros trabajos allí citados). En el caso chileno, sin embargo, no existen estudios semejantes. Por otra parte, no se conocen políticas de fomento de la participación de mujeres en la academia que se puedan haber implementado en el último tiempo, como tampoco el impacto que puedan haber tenido en la universidad las políticas públicas de equidad de género impulsadas por el gobierno.

En trabajos previos sobre mujeres en las universidades chilenas se han analizado datos por género de la evaluación y adjudicación de proyectos competitivos de investigación en Chile, y se ha evaluado el impacto de las políticas para promover la participación de las mujeres en investigación (Berríos, 2005). Un segundo trabajo (Berríos, 2007) recoge datos sobre la participación de mujeres en nombramientos de tiempo completo y en altos puestos de gestión universitaria, y muestra que ambas formas de participación han estado estancadas en la última década en Chile, evidenciando una gran brecha en este ámbito. 
Se puede señalar, de manera preliminar, que en el caso chileno las mayores diferencias en el desarrollo de carreras académicas no responderían solamente a razones de género, sino también a las variables "edad" y "disciplina". La adscripción a un determinado modelo de carrera académica también se disputa en el ámbito de las diferencias generacionales y en la cultura de las disciplinas. Este último punto, que sólo esbozamos en este artículo, esperamos desarrollarlo con mayores elementos una vez concluida la investigación sobre carrera académica.

\section{Síntesis y consideraciones finales}

Pese a la diversidad de modos de "ser académico", el sistema tiende cada vez más a un modelo homogéneo de carrera académica, instalándose como norma del modelo ideal el desarrollo de investigación y la publicación en revistas indexadas.

La idealización del modelo "investigador" es un fenómeno que se observa con gran fuerza en el sistema universitario chileno actual y se instala como el referente al que toda institución aspira para su cuerpo de profesores, independientemente si son universidades dedicadas a la investigación o a la docencia (universidades complejas vs. universidades docentes).

Aun cuando la expectativa del modelo de carrera vinculado a la investigación pueda acercar al sistema universitario hacia una gestión considerada de mayor calidad o eficiencia en el manejo de los bienes, su gran obstáculo es que no reconoce la diversidad de las instituciones y, por tanto, no puede administrarla de manera adecuada. La paradoja es que la universidad no está pensada para realizar exclusivamente investigación y sus recursos económicos no provienen mayoritariamente de esta fuente. Es la actividad docente la que genera los mayores ingresos y, no obstante ello, es la que tiene menor valoración al momento de las evaluaciones. Sin lugar a dudas, el fenómeno no se reduce a esto y, por cierto, la investigación hace más atractivas a las universidades en el mercado 
de las instituciones, lo que repercute en la captación de más y mejores estudiantes.

En este escenario, los modelos que no cumplan con este perfil tenderán a constituirse en subordinados y cada vez menos valorados. Tal como se observa, no parece que esta norma vaya a cambiar o a perder la legitimidad que actualmente le concede gran parte de la comunidad científica universitaria. Tampoco se ve factible retornar a la figura ideal de principios del siglo XX, asociada a la excelencia en la docencia y a la formación de las futuras generaciones de profesionales.

Algunas instituciones han manejado las diferencias en los modelos de carrera académica con distintas estrategias y con variados niveles de éxito; por ejemplo, estableciendo la distinción entre una carrera ordinaria (dedicada a la docencia, investigación y extensión) y la docente. Ello porque se reconoce que ciertas carreras requieren la experiencia de sus académicos en el ámbito profesional de su disciplina, como en los casos de Derecho, Medicina y Enfermería, en los cuales las exigencias al momento de evaluar se basan no en el nivel de productividad académica medida en investigaciones o publicaciones, sino en la trayectoria laboral, en el grado de experiencia adquirida "fuera de las aulas o los centros de investigación" y en el reconocimiento profesional de sus colegas.

El tema de la carrera académica es una fuente incesante de información y un buen comienzo para entender las dinámicas internas de funcionamiento de las universidades. Este artículo intenta presentar evidencia sobre las distintas realidades existentes en las universidades $y$, de ese modo, encontrar fórmulas que permitan gestionar de la manera más pertinente la diversidad que allí se encuentra.

\section{Referencias bibliográficas}

Allison, Paul and Stewart, John (1974) Productivity Differences Among Scientists: Evidence for Accumulative Advantage. American Sociological Review, 39(4), pp. 596-606. 
Altbach, Philip G.; Boyer, Ernest L. y Whitelaw, M. J. (eds.) (1994) The Academic Profession: An International Perspective. Princeton, N. J.: Carnegie Foundation for the Advancement of Teaching.

Altbach, Philip G. (ed.) (2003) The Decline of the Guru: The Academic Profession in Developing and Middle-Income Countries. New York: Palgrav Macmillan.

Berg, Helen M., y Ferber, Marianne A. (1983) Men and Women Graduate Students: Who suceeds and why? The Journal of Higher Education, 54(6), pp. 629-648.

Berríos, Paulina (2005) El sistema de prestigio en las universidades y el rol que ocupan las mujeres en el mundo académico. Calidad en la Educación, 23, pp. 349-361.

Berríos, Paulina (2007) Análisis sobre las profesoras universitarias y desafíos para la profesión académica en Chile. Calidad en la Educación, 26, pp. 39-53.

Brunner, José Joaquín (1994) Educación Superior: Chile en el contexto internacional comparado. Santiago de Chile: FLACSO.

Chavoya Peña, María Luisa (2001) Organización del trabajo y culturas académicas. Estudio de dos grupos de investigadores de la Universidad de Guadalajara. Revista Mexicana de Investigación Educativa, 6(11), pp. 79-93.

Cole, Jonathan y Cole, Stephen (1973) Social Stratification in Science. Chicago: The University of Chicago Press.

García Salord, Susana (2001) Las trayectorias académicas: de la diversidad a la heterogeneidad. Revista Mexicana de Investigación Educativa, 6(11), pp. 15-31.

Gil-Antón, Manuel (1994) Los rasgos de la diversidad: un estudio sobre los académicos mexicanos. México, D.F.: Universidad Autónoma Metropolitana.

Grediaga Kuri, Rocío (2001) Retos y condiciones de desarrollo la profesión académica en México en la última década. Revista Mexicana de Investigación Educativa, 6(11), pp. 96-117.

Kurtz-Costes, Beth, Andrews Helmke, Laura y Ulku-Steiner, Beril (2006) Gender and doctoral studies: the perceptions of PhD. Students in an American university. Gender and Education, 18(2), pp. 137-155. 
Landesmann, Monique (2001) Trayectorias académicas generacionales: constitución y diversificación del oficio académico. El caso de los bioquímicos de la Facultad de Medicina. Revista Mexicana de Investigación Educativa, 6(11), pp. 33-61.

Levy, Daniel C. (1986) Higher Education and the State in Latin America: Private Challenges to Public Dominance. Chicago: University of Chicago Press.

Merton, Robert K. (1973) The Sociology of Science. Chicago: The University of Chicago Press.

Parra Sandoval, María Cristina (2004) La percepción de los académicos sobre su participación en el gobierno universitario. Estudio de caso de cuatro universidades venezolanas. Revista Mexicana de Investigación Educativa, 9(22), pp. 665-691.

Rebufel, Viviana (2007) Participación de mujeres en fondos públicos de financiamiento de investigación científica y tecnológica. Propuestas de intervención. Santiago de Chile: CONICYT.

Stage, Frances K. y Maple, Sue A. (1996) Incompatible Goals: Narratives of Graduate Women in the Mathematics Pipeline. American Educational Research Journal, 33(1), pp. 23-51.

Stromquist, Nelly P. (ed.) (2007) The professoriate in the age of globalization Rotterdam: Sense Publishers.

Villa Lever, Lorenza (2001) El mercado académico: la incorporación, la definitividad y las promociones, pasos para una misma trayectoria de formación. Revista Mexicana de Investigación Educativa, 6(11), pp. 63-77.

Zuckerman, Harriet, Cole, Jonathan y Bruer, John (1991) The Outer Circle: Women in the Scientific Community. New York: Norton.

Recibido: 13 de mayo de 2008 Aceptado: 27 de mayo de 2008 